\title{
Editorial
}

\section{The importance of developing novel diagnostic tools for congenital metabolic disorders}

\author{
Edgard Andrade ${ }^{\mathrm{a}, *}$ and Charles Williams ${ }^{\mathrm{b}}$ \\ ${ }^{a}$ Department of Pediatrics, Division of Neurology, University of Florida College of Medicine, Gainesville, \\ FL, USA \\ ${ }^{\mathrm{b}}$ Department of Pediatrics, Division of Genetics, University of Florida College of Medicine, Gainesville, \\ FL, USA
}

Received 22 February 2012

Accepted 23 February 2012

Babies are not little adults. This frequently repeated statement implies that to develop accurate diagnostic methods to identify potentially catastrophic disorders, it is not sufficient to just wait until permanent changes have occurred and are clearly recognized by physicians in clinical practice. This is the position of many organizations that advocate on behalf of children [1]. Moreover, in the United States, the Food and Drug Administration grants approvals for diagnostic tools based on the populations in which they were studied. One of such examples is biotinidase deficiency. This autosomal recessive disorder has an estimated prevalence of 1 in 60,000 cases $[2,3]$. Most affected infants become symptomatic in the first months of life and are characterized by abnormal respiratory drive, hypotonia, failure to thrive, seborrheic dermatitis like-rash, progressive developmental delay, and myoclonic seizures. If untreated, this leads to a static-encephalopathy picture and significant motor and metal handicaps with subsequent significant morbidity and mortality and the increased costs for health care support [4]. The pathogenesis of this disease is secondary to

*Corresponding author: Edgard Andrade, University of Florida, Department of Pediatrics, Division of Neurology, PO Box 100296, Gainesville, FL 32610, USA. Tel.: +1 352273 5778; Fax: +1 352 392 9802; E-mail: andrade@peds.ufl.edu. a profound deficiency (less than $10 \%$ of enzyme activity) of biotinidase. This enzyme has ubiquitous distribution and is essential for the recycling of biotin by releasing it from any of the four holo-carboxylases: pyruvate, acetyl-CoA, propionyl-CoA, and 3-methylcrotonyl [5]. Treatment with biotin leads to a significant response and resolution of symptoms. Therefore, early diagnosis and identification of asymptomatic subjects is of the utmost importance in order to establish rapid treatment and prevent the neurological consequences of this disorder. Most laboratories typically rely on a semi-quantitative analysis for screening of this disorder in the newborn period by testing colorimetric changes of biotinidase enzyme activity in dried blood samples (DBS). However, this approach opens the possibility of impaired quality of enzyme activity in the sample, with the caveat that the mutant DNA remains relatively intact [6]. Consequently, there is a growing interest on developing more precise or adjunctive methodologies for more accurate and rapid diagnosis of this and other potentially treatable disorders. Therefore, the Lindau-Shepard et al. [7] study is a welcome addition to our understanding of diagnostic methods in the newborn period using molecular genotyping and illustrates the advantages of developing supplemental screening tools. Historically, the use of real time 
polymerase chain reaction (PCR) has been well established to detect common mutations in the biotinidase gene for early screening of every newborn in the United States [8]. In fact, a comprehensive list of 140 mutations causing biotinidase deficiency was recently reported [9]. While current practices to screen for this disorder consist of using colorimetric analysis of biotinidase enzyme activity in DBS obtained during mandated newborn screening prior to discharge from the hospital, LindauShepard et al. [7] developed a microsphere-based array, using a novel method that takes advantage of the multiplex capabilities of the Luminex platform (Luminex Corp, Austin, TX). Furthermore, the authors confirmed all of the detected genotypes in the 22 cases by DNA sequencing. Even though the assay has not been used before for the evaluation of patients with congenital errors of the metabolism such as biotinidase deficiency, certainly, it has been extensively used for microbial detection, gene expression profiling, identification of single nucleotide polymorphisms [10], human leukocyte antigen DNA typing [11], and genetic disease screening such as cystic fibrosis [12]. Furthermore, there are several examples of applications in the evaluation of human disease such as severe combined immunodeficiency $[13,14]$.

In this issue of the Journal of Pediatric Genetics, the authors tested a newly developed multiplexed microsphere-based array genotyping method for the detection of six mutations of the biotinidase gene taking advantage of the multiplexing capabilities of the Luminex platform. The DNA was extracted from DBS of biotinidase deficient newborns and the gene sequences containing the mutations of interest were amplified by PCR followed by allele specific primer extension. Subsequently, the genotypes were verified by sequencing and the results were compared an found concordant for all 6 mutations tested in the 22 known biotinidase deficient samples previously identified by the authors using DBS biotinidase enzyme screening. The process was replicated several times to confirm accuracy and the results consistently showed identical genotypes for all tested samples. This feasibility study revealed several advantages: 1) lower amount of genomic DNA needed as compared with non-multiplexed genomic methods, 2) reduced amount of processing time, 3) small amount of reagent required due to the application of PCR amplification techniques on the genomic DNA, 4) easily tailored process depending on the laboratory needs by adding or removing microsphere sets, 5) customizable panels of analysis according to geographical mutation prevalence.
A rapid diagnostic tool with the ability to safely and efficiently establish the diagnosis of any neurometabolic disorder such as biotinidase deficiency in the first days of life would be an important addition to the diagnostic armamentarium available to screen newborns with a potentially treatable disorder.

Because of the many different mutations associated with biotinidase deficiency, it is clear that DBS screening for biotinidase enzyme activity will remain the primary method for identifying presumptive positives and that follow up blood enzyme analysis will remain the gold standard for confirmation of both partial and profound biotinidase deficiency. However, the addition of a molecular genotyping component to the newborn screening event, as presented in this report, could provide important assistance in the initial evaluation of presumptive positives. For example, the demonstration of a $\mathrm{D} 444 \mathrm{H}$ mutation without presence of the A171T mutation provides very high probability that the newborn will have partial and not profound enzymatic activity [15]. This is because the D444H mutation alone without its A171T mutation in cis orientation allows for only moderate reduction in enzyme activity. Alternatively, the demonstration of two severe mutations creates high probability that the infant has profound biotinidase deficiency. This type of information can help physicians responsible for following up on presumptive positive results and for subsequently orchestrating referrals of such infants to neurologists or other individuals for further evaluation and treatment.

\section{References}

[1] Watson MS, Mann MY, Lloyd-Puryear MA, Rinaldo PR, Howell RR. Newborn screening: toward a uniform screening panel system-executive summary. Pediatrics 2006;117(5 Pt 2): 296-307.

[2] Rezvani I, Rosenblatt D. Valine, leucine, isoleucin and related organic acidemias. In: Behrman R, Kliegman R, Jenson H, editors. Textbook of pediatrics. 16th ed. Philadelphia: WB Saunders; 2000. p. 354-62.

[3] Ogundele MO. Question 2. What is the incidence of biotin deficiency in preschool children with global developmental delay? Arch Dis Child 2011;96(9):895-7.

[4] Wolf B. The neurology of biotinidase deficiency. Mol Genet Metab 2011;104(1-2):27-34.

[5] Pindolia K, Jordan M, Guo C, Matthews N, Mock DM, Strovel E, et al. Development and characterization of a mouse with profound biotinidase deficiency: a biotin-responsive neurocutaneous disorder. Mol Genet Metab 2011;102(2):161-9.

[6] Adam BW, Hall EM, Sternberg M, Lim TH, Flores SR, O'Brien S, et al. The stability of markers in dried-blood spots for recommended newborn screening disorders in the United States. Clin Biochem 2011;44(17-18):1445-50. 
[7] Lindau-Shepard B, Janika DK, Passb KA. A microspherebased assay for mutation analysis of the biotinidase gene using dried blood spots. J Pediatr Genet 2012;1(3):153-160.

[8] Dobrowolski SF, Angeletti J, Banas RA, Naylor EW. Real time PCR assays to detect common mutations in the biotinidase gene and application of mutational analysis to newborn screening for biotinidase deficiency. Mol Genet Metab 2003;78(2):100-7.

[9] Pindolia K, Jordan M, Wolf B. Analysis of mutations causing biotinidase deficiency. Hum Mutat 2010;31(9):983-91.

[10] Ivanova M, Ruiqinq J, Kawai S, Matsushita M, Ochiai N, Maruya E, et al. IL-6 SNP diversity among four ethnic groups as revealed by bead-based liquid array profiling. Int J Immunogenet 2011;38(1):17-20.

[11] Ivanova M, Ruiqinq J, Matsushita M, Oqawa T, Kawai S, Ochiai N, et al. MBL2 single nucleotide polymorphism diversity among four ethnic groups as revealed by a bead-based liquid array profiling. Hum Immunol 2008;69(12):877-84.
[12] Dunbar SA. Applications of Luminex xMAP technology for rapid, high-throughput multiplexed nucleic acid detection. Clin Chim Acta 2006;363(1-2):71-82.

[13] Janik DK, Lindau-Shepard B, Comeau AM, Pass KA. A multiplex immunoassay using the Guthrie specimen to detect T-cell deficiencies including severe combined immunodeficiency disease. Clin Chem 2010;56(9):1460-5.

[14] Bortolin S, Black M, Modi H, Boszko I, Kobler D, Fieldhouse $\mathrm{D}$, et al. Analytical validation of the tag-it high-throughput microsphere-based universal array genotyping platform: application to the multiplex detection of a panel of thrombophiliaassociated single-nucleotide polymorphisms. Clin Chem 2004; 50:2028-36.

[15] Norrgard KJ, Pomponio RJ, Swango KL, Hymes J, Reynolds T, Buck GA, et al. Double mutation (A171T) and (D444H) is a common cause of profound biotinidase deficiency in children ascertained by newborn screening in the United States. Hum Mutat 1998;11(5):410-23. 